\title{
I PRINCIPI DELLA DINAMICA DI TARTAGLIA E CARDANO NELLE CINQUECENTINE DELLISTITUTO LOMBARDO
}

\author{
Nota del m.e. CARLO ENRICO BOTTANI (*)
}

(Adunanza del 5 giugno 2014)

SunTO. - La biblioteca dell'Istituto Lombardo possiede due cinquecentine contenenti capitoli di argomento fisico: La nova scientia di Niccolò Tartaglia stampato In Venetia: per Nicolò de Bascarini a istantia de l'Autore, 1550 e il De rerum varietate di Girolamo Cardano stampato Basileae: per Sebastianum Henricpetri, 1581. Assieme al De Subtilitate di Girolamo Cardano, vengono in queste opere trattati i principi generali della dinamica e, con grande dettaglio, la traiettoria di un proiettile sparato da una arma da fuoco. Anche se i principi generali del moto sono ancora di impronta aristotelica, l'attenzione ai fatti sperimentali ed un uso spregiudicato di argomenti eterogenei di spiegazione, finalizzati al raggiungimento di una capacità predittiva con scopi eminentemente pratici, permettono di identificare in Tartaglia e in Cardano veri precursori della moderna balistica. La rivisitazione cardaniana, oggi insostenibile, del ruolo di forza motrice dell'aria, richiesto dall'assenza della legge d'inerzia nella dinamica aristotelica, e del concomitante ruolo di forza resistente, suggerisce lo spunto per una considerazione di principio sulla persistenze mancanza di una deduzione microscopica rigorosa del secondo principio della termodinamica e, in genere, sull'origine delle forze dette dissipative.

$* * *$

ABSTRACT. - The library of Istituto Lombardo owns two volumes of XVI century (cinquecentine) including physical sections. La nova scientia by Niccolò Tartaglia printed In Venetia: per Nicolò de Bascarini a istantia de l'Autore, 1550 and the De re-

Milano, Italy.

E-mail: carlo.bottani@polimi.it 
rum varietate by Girolamo Cardano printed Basileae: per Sebastianum Henricpetri, 1581. Together with the De Subtilitate by Girolamo Cardano, these books contain the general laws of dynamics and, in great detail, a description of the trajectory of a projectile fired from a firearm. Though the general principles are still of Aristotelian derivation, a new attention to experimental facts together with an unconvential pragmatic use of etherogeneous explanation arguments, justified by an essentially practical goal of predictive nature, induce one to identify both Tartaglia and Cardano as true precursors of modern ballistics. Furthermore, the way Cardano revisits the role of driving force played by the projectile-air interaction, following a modified idea of Aristotle due to the lacking of an inertia law in his mechanics, and the concomitant need that air produces also a resisting force, reminds us that, so far, a rigorous microscopic derivation of the exclusively dissipative nature of this force has not been achieved yet as well as a rigorous microscopic derivation of the second principle of thermodynamics.

\author{
"Ma a causa di una proposizione, che è vera, cioè \\ che ogni corpo che muove è mosso, si pone ora \\ lo stesso interrogativo per l'aria, e perciò se \\ l'aria muove la pietra lanciata, essa è mossa; \\ quindi l'aria è mossa da qualcosa, e così ci \\ ritroviamo come nel caso della pietra. Dicono \\ che l'aria, essendo leggera, sia mossa dalla sua forma" \\ Traduzione da De subtilitate di Girolamo Cardano \\ del dott. Elio Nenci (comunicazione privata)
}

\title{
1. INTRODUZIONE
}

La data di nascita della fisica moderna non è, ovviamente, determinabile con esattezza. Si arriva all'attuale stato della scienza fisica attraverso un processo con accelerazioni e decelerazioni che trova in Galileo Galilei (1564-1642) una nuova consapevolezza e un nuovo uso della sperimentazione che condurranno a geniali, anche se parziali, intuizioni per una dinamica rinnovata e, in Isaac Newton (16421727), un punto di svolta di eccezionale rilevanza. Personalmente ritengo che non solo la fisica ma anche, in generale, la scienza moderna diventino pienamente consapevoli del nuovo modus operandi con quel celeberrimo "Hypotheses non fingo" nello Scolium generale dei Principia (seconda edizione del 1713). Anthony Kenny, in Nuova Storia della Filosofia Occidentale (Torino, Einaudi, 2012), in quattro volumi, dichiara nel capitolo Fisica del terzo volume, dedicato ai seco- 
li XVI-XVII-XVIII, di non aver voluto includere questo capitolo nel quarto volume sulla filosofia contemporanea in quanto, a partire da Newton, la Fisica si è guadagnata il ruolo di scienza matura, degna di una trattazione separata da quella della filosofia. E' curioso, a tal proposito, che il titolo della rivoluzionaria opera newtoniana sia ancora Philosophiae naturalis principia mathematica! Per i nostri scopi risulta comunque chiaro che il XVI secolo, cui qui rivolgiamo il nostro interesse riguardo alle cinquecentine dell'Istituto Lombardo, va considerato un periodo di transizione in cui si manifestano numerose embrionali anticipazioni della fisica moderna, soprattutto per quanto riguarda la dinamica, ma non solo. Mi preme tuttavia rilevare che anticipazioni di epoca medievale, talora impressionanti per precisione e potenziale capacità predittiva, di alcuni capitoli della fisica attuale, sono state rese disponibili all'indagine storica solo in tempi recenti o recentissimi. Ne cito solamente due. Thomas Bradwardine (12901349), arcivescovo di Canterbury, membro del Merton College di Oxford, contemporaneo di Guglielmo di Occam, uno degli Oxford Calculators, nel De proportionibus velocitatum in motibus scoprì le leggi del moto uniformemente accelerato. Il fisico matematico e storico della scienza Clifford Truesdell (già membro straniero dell'Istituto Lombardo) scrisse [1, pag. 30]: "The now published sources prove to us, beyond contention, that the main kinematical properties of uniformly accelerated motions, still attributed to Galileo in the physics texts, were discovered and proved by scholars of Merton college.... In principle, the qualities of Greek physics were replaced, at least for motions, by the numerical quantities that have ruled Western science ever since. The work was quickly diffused into France, Italy, and other parts of Europe. Almost immediately, Giovanni di Casale and Nicole Oresme found how to represent the results by geometrical graphs introducing the connection between geometry and the physical world that became a second characteristic habit of Western thought ...". Ritengo ancora più impressionante la recente scoperta del ruolo profetico di Roberto Grossatesta (1175-1253), vescovo di Lincoln, riguardo ad argomenti di cosmologia e fisica del colore. I contributi del vescovo alla scienza sono ben noti da molto tempo, ma non era stato adeguatamente riconosciuto l'aspetto assolutamente moderno e, potenzialmente, quantitativo e predittivo di due sue idee contenute nel De luce. Ringrazio il caro amico prof. Brian K. Tanner, professore emerito di fisica nell'università di Durham (UK), per avermi segnalato una serie di lavori 
recenti [2] che hanno messo il luce un aspetto del contributo del Grossatesta che non esito a definire attuale. Probabilmente lo studio della scienza medievale ci riserverà ancora non poche sorprese.

\section{LA FISICA NEL CINQUECENTO: OPERE PRINCIPALI}

La storia della scienza [3] riconosce i seguenti come i principali contributi del cinquecento alla fisica:

- Buonamici Francesco (1530-1603) De Motu (conosciuto e respinto da Galileo in quanto ancora completamente legato alla dinamica aristotelica).

- Cardano Girolamo (1501-1576) De rerum varietate (ce ne occupiamo direttamente qui) De subtilitate (ce ne occupiamo direttamente qui).

- Tartaglia (Fontana) Niccolò (1499-1557) La nova scientia (ce ne occupiamo direttamente qui).

- Benedetti Giambattista (1530-1590) Diversarum speculationum mathematicarum et physicarum liber (anticipa in qualche misura il principio d'inerzia rispetto a Galileo e, soprattutto, a Cartesio).

- Maurolico Francesco (1494-1575) Photismi de lumine et umbra Diaphana (importanti opere di ottica pubblicate postume nel 1611. Galileo non le aveva lette: ciò spiega la relativa ignoranza di Galileo riguardo all'ottica e l'impossibilità da parte sua di comprendere i limiti tecnici del suo cannocchiale).

- Commandino Federico (1509-1575) Horologiorum descriptio (studi di statica).

- Del Monte Guidobaldo (1545-1607) Mechanicorum liber (corrispondente di Galileo).

- Stevin Simon (1548-1620) De Beghinselen der Weegconst (Principi di statica e idrostatica).

\section{LE CINQUECENTINE DI CONTENUTO FISICO DELL'ISTITUTO LOMBARDO E Il De SubTilitate}

La biblioteca dell'Istituto Lombardo possiede due di queste opere: La nova scientia di Niccolò Tartaglia Stampato In Venetia: per Nicolò de Bascarini a istantia de l'Autore, 1550 (Fondo Arnaldo 
Masotti) e il De rerum varietate di Girolamo Cardano stampato Basileae: per Sebastianum Henricpetri, 1581(Fondo Luigi Belloni), di cui sono mostrate di seguito due immagini (Fig. 1).

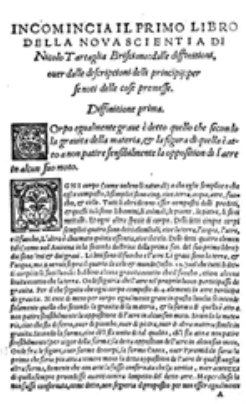

a

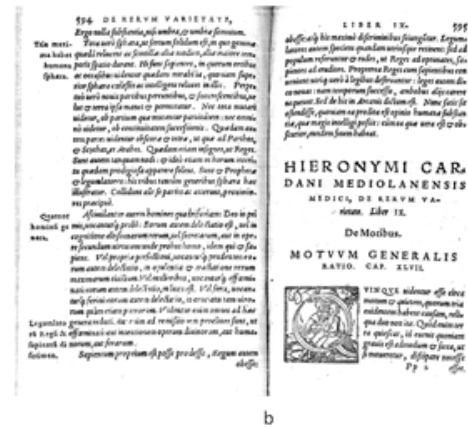

Fig. 1 - a) L'incipit della Nova Scientia; b) due pagine del De Rerum Varietate.

\section{L'EREDITÀ ARISTOTELICA NEI PRINCIPI DELLA DINAMICA DI TARTAGLIA E CARDANO}

Tartaglia e Cardano si basano su principi generali del moto di impronta ancora sostanzialmente aristotelica, introducendo tuttavia alcune significative, anche se marginali, variazioni e innovazioni. Per comodità del lettore, riassumiamo qui i punti base della dinamica di Aristotele.

- Il cambiamento di posizione è solo uno dei possibili fatti meccanici: il moto locale dei corpi è il quarto tra i cambiamenti possibili nel mondo sublunare: questi cambiamenti possono essere di sostanza, di qualità, di quantità e di posizione.

- Il vuoto non esiste (si consideri la posizione radicalmente diversa di Newton [4] e il ritorno all'antico dell'attuale QFT-Quantum Field Theory [5]).

- I possibili tipi di moto sublunare sono due: naturale (per esempio la caduta di un grave) e violento (per esempio il moto iniziale di una freccia scoccata da un arco).

- Non c'è legge d'inerzia: il moto richiede sempre una causa (talora difficile da evidenziare per i moti naturali). 
- Il moto naturale dei gravi (verso il centro della terra) avviene con velocità media proporzionale al peso e inversamente proporzionale alla densità del mezzo.

- Nel moto violento la velocità è proporzionale alla forza motrice e inversamente proporzionale alla resistenza del mezzo. La dinamica dell'aria fornisce sia la forza motrice (dopo il primo impulso) sia la forza resistente. Vedi di seguito le connessioni con la moderna teoria cinetica microscopica e il secondo principio della termodinamica.

- E' in buon accordo con molti dati sperimentali, soprattutto per i moti stazionari in presenza di resistenza viscosa.

Non affronterò analiticamente i punti in cui Tartaglia e, soprattutto, Cardano si discostano, nel merito, dalle leggi aristoteliche generali. Basti qui ricordare che entrambi si basano su Aristotele ma con una certa autonomia di pensiero e con un attenzione importante ai fatti sperimentali:

- utilizzano in modo nuovo un concetto precedente di moto di tipo misto (in parte violento, in parte naturale), introdotto empiricamente per meglio salvare i fenomeni e, per la prima volta, interessati alla precisa forma geometrica della traiettoria relativa a questo tipo di moto.

- Tartaglia, con una taglio che diremmo fisico-matematico, introduce originali postulati ad hoc, alcuni mutuati dagli elementi di Euclide, per esempio: Lo istante è quello che non ha parte (Il ponto è quello che non ha parte).

- Cardano si discosta dall'interpretazione corrente del filosofo nella descrizione dettagliata dell'interazione con l'aria di un corpo in moto violento. Egli ritiene che la potenza motrice dell'aria nei confronti di un proiettile non sia stata compresa: solo lui, Cardano, ha capito cosa Aristotele voleva intendere e, comunque, il filosofo deve essere parzialmente corretto.

Del resto già Filopono, un autore del VI secolo dell'era cristiana, aveva criticato Aristotele su questo punto [6].

Qui mi concentrerò esclusivamente sulle innovazioni introdotte dai nostri due autori nel caso particolare del moto di un proiettile, caso trattato in estremo dettaglio da entrambi nelle due cinquecentine dell'Istituto. Va però detto subito che Cardano tratta la balistica con 
dettaglio ancora maggiore in un'altra opera che l'Istituto Lombardo non possiede: il De Subtilitate, di cui è stata pubblicata a cura del dott. Elio Nenci un'edizione critica parziale [7]. Uno degli esemplari più antichi del testo, stampato da Johann Petrejus, Nürnberg: 1550, si trova a Milano (Braidense, coll. D XIV 10, 948). Grazie alla cortesia del dott. Nenci ho potuto leggere in anteprima la sua traduzione italiana inedita del capitolo del De Subtilitate che tratta del moto di un proiettile. Un esempio della bella traduzione del dott. Nenci è stato da me utilizzato nella citazione di apertura. Ho così potuto integrare le informazione contenute nel De Rerum Varietate e completare il confronto con la visione di Tartaglia.

\section{Il MOTO DI UN PROIETTILE IN TARTAgLia E CARDANO}

Nel cinquecento la balistica assume sempre di più i tratti di una scienza: le motivazioni pratiche, essenzialmente militari, sono ovvie. Tartaglia e Cardano si preoccupano degli aspetti sperimentali della traiettoria di un proiettile sparato da un arma da fuoco. Si rendono entrambi conto che i principi della fisica aristotelica non permettono di descrivere adeguatamente la forma dettagliata di tutta la traiettoria. Assumono allora entrambi un atteggiamento estremamente pragmatico e adottano una spiegazione non unitaria del fenomeno. La traiettoria è divisa in tre tratti. Il moto iniziale, rettilineo, è ben spiegato come moto violento, in accordo con Aristotele. Come accennavo, Cardano si discosta dalla descrizione classica nell'analisi particolareggiata dell'interazione con l'aria (che fornisce anche la forza resistente). Il lettore interessato potrà trovare i dettagli nel De Subtilitate. Più avanti riprenderò brevemente questo aspetto dal punto di vista della cinetica fisica moderna. Il terzo e ultimo tratto della traiettoria viene assunto come un moto naturale verticale di caduta. In questo entrambi gli autori abbandonano, curiosamente, il tentativo di tenere conto della traiettoria reale che non ha affatto questa forma: si tratta dell'aspetto più conservatore e meno attuale. Il maggiore elemento di novità sta nella descrizione del secondo tratto (intermedio) della traiettoria. Empiricamente si assiste ad un moto curvilineo con la concavità verso il basso. Tartaglia e Cardano non possono trovarne la forma né una spiegazione semplice nella fisica del filosofo. Ricorrono allora, in modo puramente fenomenologico, ad un raccordo del primo e del 
terzo tratto con un arco di curva che assomigli a quello effettivamente osservato (Fig. 2). Non sono più interessati a dedurre il moto da principi generali, ma alla costruzione di una descrizione predittiva di utilità pratica. La differenza sta nella scelta della forma del tratto di raccordo. Tartaglia adotta un arco di cerchio. Non conosceva il trattato sulle coniche di Apollonio: non poteva fare di meglio. Cardano, dimostrando maggiore aderenza alla forma reale e, forse, guidato da un misterioso intuito, assume invece un arco di parabola. La nuova meccanica del seicento gli darà ragione, anche se solo nel caso in cui si trascuri la resistenza dell'aria. Paradossalmente il raccordo con il primo e il terzo tratto viene rappresentato graficamente con punti angolosi, imperfezione assente nei disegni di Tartaglia. Potremmo allora riassumere. Tartaglia si è posto il problema della forma della traiettoria usando anche esperimenti che lo hanno costretto all'introduzione di un moto misto di raccordo. Così anche Cardano, che ha però introdotto l'idea della parabola, più vicina alla descrizione corretta. Guidobaldo Del Monte estenderà la somiglianza della parabola a tutta la traiettoria eguagliando i tratti ascendente e discendente. Infine Galileo - partendo dall'assunto errato dell'uguaglianza di catenaria e parabola - assimilerà l'intera traiettoria a quest'ultima curva. Sarà tuttavia Bonaventura Cavalieri (1598-1647) a fornire per primo la dimostrazione esatta nell'opera Lo specchio ustorio, precedendo Galileo.

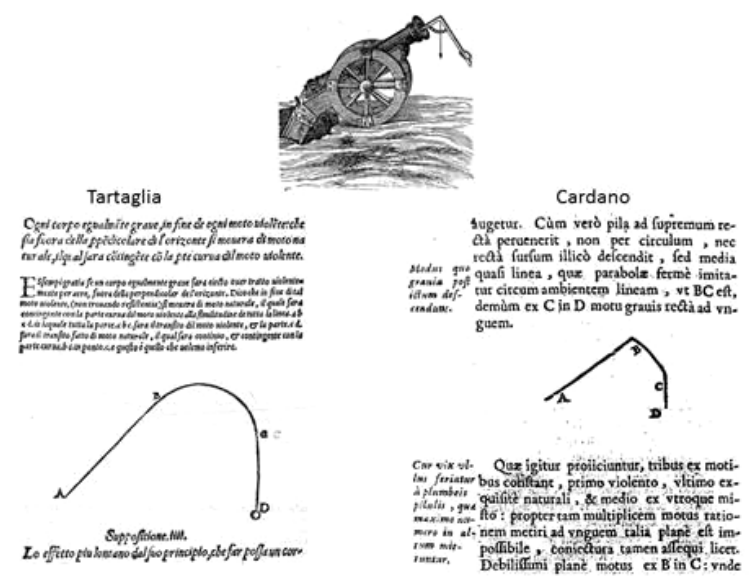

Fig. 2 - Una bombarda e la traiettoria del proiettile nella Nova Scientia (a sinistra); la traiettoria del proiettile nel De Subtilitate (a destra). 


\section{LA FORZA MOTRICE RISULTANTE DALL'INTERAZIONE ARIA-PROIETTILE SECONDO ARISTOTELE E LA DEDUZIONE MICROSCOPICA DEL SECONDO PRINCIPIO DELLA TERMODINAMICA}

Come abbiamo già accennato un uso inedito, oggi si direbbe opportunistico, del vecchio concetto di moto misto, per salvare i fenomeni, soprattutto la forma della parte centrale della traiettoria, innestato nello schema logico deduttivo della precedente dinamica aristotelica, costituisce un importante elemento di novità. Si accetta una spiegazione composta da due parti, tra loro disgiunte e incoerenti, che nell'insieme permette una maggiore capacità predittiva, rinunciando ad una giustificazione unitaria. Il ruolo dell'interazione del proiettile con l'aria, che produce, secondo Tartaglia e Cardano, sia forza motrice, subito dopo l'esplosione della polvere da sparo, sia forza resistente, anche se descritto da entrambi gli autori con argomentazioni aristoteliche modificate, oggi insostenibili, ci ricorda inevitabilmente che la moderna cinetica fisica non ha completamente risolto il complesso problema del moto di un corpo immerso in un fluido viscoso dal punto di vista molecolare dettagliato. Si tratta di un caso particolare del formidabile problema della derivazione microscopica rigorosa dell'irreversibilità intrinseca di tutti i fenomeni macroscopici, da cui seguono i concetti di dissipazione dell'energia e di forze dissipative, connessi con quello di produzione di entropia (mai negativa) e della freccia del tempo che punta sempre dal passato verso il futuro. Boltzmann credette di aver risolto il problema con la sua dimostrazione del cosiddetto teorema $\mathrm{H}$, cui furono opposte significative obiezioni, basate sul teorema del ritorno di Poincaré e sulla reversibilità temporale di tutte le leggi di forza microscopiche. Per quanto riguarda il primo, si tratta del fatto che un sistema dinamico, pur di aspettare un tempo sufficiente, ritorna sempre in un intorno infinitesimo delle condizioni iniziali. Per quanto riguarda il secondo aspetto, la difficoltà risiede nella proprietà di invarianza rispetto all'inversione del tempo $(t \rightarrow-t)$ di tutte le dinamiche microscopiche, classiche e quantistiche. Per chi voglia approfondire tale affascinante tema, consigliamo la lettura di alcune belle pagine di un libro di Carlo Cercignani (già membro effettivo del nostro Istituto): vedi [8], pagg.140-141, sulla fallacia del teorema $\mathrm{H}$ di Boltzmann per particolari insiemi di condizioni iniziali. Il tema è: il secondo principio della termodinamica non è derivabile rigorosamente dalle leggi dinamiche microscopiche. Nel caso del moto Browniano di un colloide (oggi si direbbe di una nanoparticella) il problema è stato affrontato da Einstein e da 
Smoluchowski, apprendo la strada alla teoria dei rapporti tra fluttuazioni e dissipazione [9]. Nel caso di un corpo macroscopico che si muove in un fluido la deduzione microscopica rigorosa del moto medio, in particolare della forza media resistente, descritta oggi dalla legge empirica di Stokes, in accordo con i dati sperimentali, ${ }^{1}{ }^{\mathrm{e}}$ ancora un problema aperto. Aristotele aveva bisogno di invocare anche il ruolo di forza motrice dovuta all'interazione del proietto con l'aria: la sua meccanica difettava della legge d'inerzia. La spiegazione aristotelica, anche nella forma modificata da Cardano, è oggi assolutamente inaccettabile. Tuttavia: è anche tale la mera idea che l'effetto medio di innumerevoli urti molecolori possa produrre, in linea di principio, una forza motrice, invece di una forza resistente? Possa cioè violare il secondo principio della termodinamica? Il singolo urto microscopico può produrre un'accelerazione equiversa alla velocità istantanea appena precedente l'urto stesso. Perché l'effetto medio di tutti gli urti in un intervallo di tempo finito è sempre equivalente ad una forza resistente (dissipativa) opposta alla velocità? Nel nostro caso, per quanto praticamente irrealizzabile a causa di probabilità estremamente basse (così risponderebbe Boltzmann e così testimoniano i fatti sperimentali), non è, in teoria, completamente inconcepibile un mezzo fluido dove la produzione di entropia sia negativa. Ciò, non violando alcuna legge microscopica nota, comporterebbe una viscosità negativa [10]: se così fosse la legge di Stokes (Fig. 3) si trasformerebbe in una forza motrice.

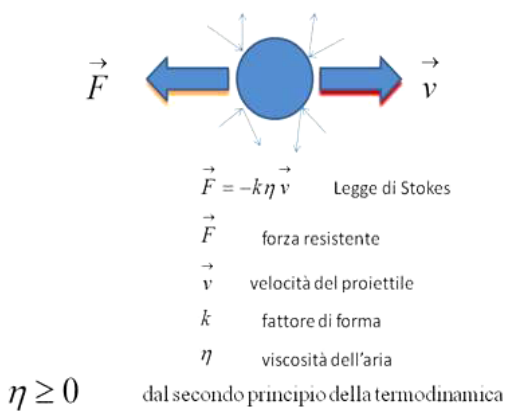

Fig. 3 - Illustrazione grafica della forza di Stokes. Sono schematicamente illustrati quattro eventi corrispondenti a urti con molecole del fluido.

1 Solo nel caso di fluido in quiete e di basse velocità del corpo che si muove nel fluido. Non è il caso normalmente considerato nella balistica. Vedi le conclusioni. 
Si tratta, naturalmente, di una pura suggestione, anche anacronistica: nulla era più estraneo ad Aristotele quanto l'idea di una struttura atomica della materia. Idea estranea ad Aristotele ma non all'antichità tutta: Leucippo, Democrito e Lucrezio insegnano...

\section{CONCLUSIONI}

Le due cinquecentine dell'Istituto Lombardo, di Tartaglia e Cardano, di cui si è qui discusso limitatamente agli aspetti fisici riguardanti il moto di un proiettile, completando l'analisi con la corrispondente parte del De Subtilitate, sempre di Cardano, consentono, da un lato, di considerare Tartaglia come il padre della moderna balistica e, dall'altro, Cardano come colui che, per primo, anticipa, per una parte della traiettoria di un proiettile, la forma parabolica che la nuova meccanica newtoniana assegnerà a tutta la traiettoria nel caso limite di resistenza dell'aria trascurabile. Il principale aspetto innovativo in Tartaglia è l'uso dei fatti sperimentali assieme ai vecchi principi aristotelici, generando una spiegazione concettualmente non unitaria ma assai efficace ai fini predittivi, per esempio dell'alzo della bocca da fuoco che produce la gittata massima. Cardano, oltre ad indovinare la forma parabolica di un tratto di traiettoria, senza giustificazione alcuna, si dimostra intellettualmente più indipendente da Aristotele nella descrizione dell'interazione con l'aria. Interazione, quest'ultima, non suscettibile di una descrizione semplice, neppure oggi. Per un interessante rassegna delle edizioni cardaniane del CNR si veda il riferimento [11]. La storia della balistica [12, pag. 971] ci insegna che la forza resistente non è descritta adeguatamente dalla legge di Stokes. Essa può, generalmente, dipendere da una potenza n della velocità (con $\mathrm{n}$ diverso da 1). Eulero fu il primo a ridurre alle quadrature le equazioni del moto in questo caso. Oggi il problema è risolto numericamente assumendo leggi di resistenza empiriche che devono tenere conto anche della dinamica atmosferica e del moto di rotazione del proiettile prodotto dalla rigatura delle canne da fuoco. La bella e semplice parabola, che qualunque moderno libro di testo di Meccanica deriva nel vuoto, ha quindi, più che altro, un valore didattico introduttivo ad un problema ben più complesso, così complesso da valorizzare maggiormente i contributi contenuti nelle opere di Tartaglia e Cardano, considerando i mezzi concettuali e tecnici a loro disposizione. 


\section{RINGRAZIAMENTI}

Ringrazio sentitamente il prof. Giannantonio Sacchi-Landriani per avere stimolato il mio interesse all'argomento e il prof. Enrico Isacco Rambaldi per avermi segnalato gli studi su Cardano del dott. Elio Nenci; entrambi per aver letto in anteprima il manoscritto di questa nota. Devo molta riconoscenza al dott. Elio Nenci per aver redatto in tempi brevissimi una bella traduzione in italiano del capitolo sul moto del De Subtilitate e per l'estrema cortesia con cui me l'ha resa disponibile.

\section{BIBLIOGRAFIA}

[1] Clifford Truesdell, Essays in The History of Mechanics, Springer-Verlag, New York, 1968.

[2] Richard G. Bower et al., Proc. R. Soc. A, 2014: 470, 20140025; Tom C.B. McLeish et al., Nature, 2014: 507, 162; Hannah E. Smithson et al., J. Opt. Soc. Am. A, 2014: 31, A341.

[3] Marie Boas, Il Rinascimento scientifico: 1450-1630, Feltrinelli, 1973; Paolo Rossi, La nascita della scienza moderna in Europa, Laterza, 1997; H. Butterfield, Le origini della scienza moderna, Il Mulino, 1962.

[4] Alexandre Koyré, Studi Newtoniani, Einaudi, 1983.

[5] Matthew D. Schwartz, Quantum Field theory and the Standard Model, Cambridge University Press, 2014.

[6] Ernst Mach, La Meccanica nel suo sviluppo storico-critico, Universale Scientifica Boringhieri, 1977.

[7] Elio Nenci (a cura di), Girolamo Cardano, De Subtilitate, Libri I-VII. Tomo I, Franco Angeli, Milano, 2004.

[8] Carlo Cercignani, The Boltzmann equation and ist Applications, Springer Verlag, 1988.

[9] Albert Einstein, Über die von der molekularkinetischen Theorie der Wärme geforderte Bewegung von in ruhenden Flüssigkeiten suspendierten Teilche, Annalen der Physik, 1905: 322 (8), 549-560; Marian von Smoluchowski, Zur kinetischen Theorie der Brownschen Molekularbewegung und der Suspensionen, 1906: Annalen der Physik 326 (14), 756-780.

[10] Lev D. Landau, Evgenij M. Lifshitz, Fluid Mechanics 2-nd ed., Course of Theoretical Physics vol. 6, Pergamon Press, 1987.

[11] Enrico I. Rambaldi, Rivista di Storia della Filosofia, 2010: 4, 745-773.

[12] Attilio Lazzarini, voce Balistica nell'Enciclopedia Italiana, vol. V, Istituto dell'Enciclopedia Italiana fondata da Giovanni Treccani, Roma, 1949. 